Gérald van de Werve Borhane Annabi Alfred Berteloot Jean-François St-Denis Hubert Vidal

\section{ADRESSES}

G. van de Werve : directeur du département de nutrition, Groupe de recherche en transport membranaire.

B. Annabi : étudiant au doctorat, département de biochimie.

A. Bertheloot : professeur agrégé au département de physiologie, Groupe de recherche en transport membranaire.

J.-F. St-Denis : étudiant au doctorat, département de biochimie, faculté de médecine, Université de Montréal, C.P. 6128 succursale A, Montréal H3C 3J7 Canada.

H. Vidal : chargé de recherche à l'Inserm, Inserm

$U .197$, faculté de médecine Alexis-Carrel,

8 , rue Guillaume-Paradin, 69372 Lyon

Cedex 08, France.

$\mathrm{m} / \mathrm{s} n^{\circ} 5$ vol. 9 , mai 93

\title{
Le système glucose 6-phosphatase hépatique : composantes, propriétés cinétiques, régulation et déficit
}

Le glucose 6-phosphatase (G6Pase) catalyse la dernière étape enzymatique menant à la libération de glucose par le foie. Elle est donc indispensable au maintien de la glycémie. Sa situation stratégique au carrefour des voies métaboliques de la néoglucogenèse, de la glycogénolyse et de la glycolyse ainsi que la sévérité des troubles métaboliques qu'entraînent ses différentes formes de déficit génétique (glycogénoses de type 1) font de l'étude moléculaire du fonctionnement de ce système enzymatique une étape cruciale pour la compréhension du métabolisme du glucose in vivo. Dans cet article, deux types d'organisation moléculaire du système G6Pase sont examinés, le modèle conformationnel et le modèle de transport du substrat, ainsi que la régulation hormonale de la G6Pase et les bases moléculaires de son déficit génétique. Nos résultats apportent en faveur du premier modèle des arguments fondés sur les propriétés cinétiques et la spécificité de l'enzyme pour son substrat. Un transport unidirectionnel du glucose produit lors de l'hydrolyse du G6P, a en outre été mis en évidence dans les microsomes.

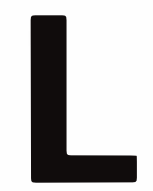
a glucose 6-phosphatase (G6Pase) [D-glucose 6-phosphate phosphohydrolase, EC 3.1.3.9] est l'enzyme qui catalyse la dernière étape de la production de glucose dans le foie et le cortex rénal, en hydrolysant le glucose 6-phosphate produit tant par la néoglucogenèse que par la dégradation des réserves de glycogène [1]. La G6Pase est cons- tituée d'un complexe multipeptidique intégré dans la membrane du réticulum endoplasmique. Cet article examinera seulement l'activité phosphohydrolase de la G6Pase, fonction qui joue un rôle primordial dans le contrôle de l'homéostase du glucose. La compartimentalisation de la G6Pase permet d'isoler l'enzyme de façon simple en préparant une fraction microsomale, enrichie en vésicu- 


\section{RÉFÉRENCES}

1. Nordlie RC, Sukalski K. Multifunctional glucose-6-phosphatase : a critical review. In : Martonosi AN, ed. The enzymes of biological membranes. Vol. 2. New York: Plenum Press, 1985 ; 349-98.

2. Arion $\mathrm{WJ}, \mathrm{Wallin} \mathrm{BK}$, Lange $\mathrm{AJ}, \mathrm{Bal}$ las LM. On the involvment of a glucose 6-phosphate transport system in the function of microsomal glucose 6-phosphatase. Mol Cell Biochem $1975 ; 6$ : 75-83.

3. Fulceri R, Bellomo G, Gamberucci A, Scott H, Burchell A, Benedetti A. Permeability of rat liver microsomal membrane to glucose 6-phosphate. Biochem J $1992 ; 286$ 813-7.

4. Nilsson OS, Arion WJ, De Pierre JW, Dallner G, Ernster L. Evidence for the involvement of a glucose-6-phosphate carrier in microsomal glucose-6-phosphatase activity. Eur J Biochem 1978; 82 : 627-34.

5. Stetten MR, Burnett FF. Some properties of variously activated microsomal glucose-6-phosphatase, inorganic pyrophosphatase and inorganic pyrophosphate-glucose phosphotransferase. Shift in $\mathrm{pH}$ optimum. Biochem Biophys Acta 1967 ; 139 : 138-47.

6. Zakim D, Dannenberg A. Thermal stability of microsomal glucose-6-phosphatase. J Biol Chem 1990; 265 : 201-8.

7. Schulze H-U, Nolte B, Kannler R. Evidence for changes in the conformational status of rat liver microsomal glucose-6-phosphate : phosphohydrolase during detergent-dependent membrane modification. Effet of $p$-mercuribenzoate and organomercurial agarose gel on glucose-6-phosphatase of native and detergent-modified microsomes. $J$ Biol Chem 1986 ; $261: 16571-8$

8. Ness GC, Sukalski KA, Sample CE, Pendleton LC, McCreery MJ, Nordlie RC. Radiation inactivation analysis of rat liver microsomal glucose-6-phosphatase. J Biol Chem 1989 ; 264 : 7111-4.

9. Speth M, Schulze H-U. The purification of a detergent-soluble glucose-6-phosphatase from rat liver. Eur $J$ Biochem 1992 ; 208 : 643-50.

10. Feldman F, Butler LG. Detection and characterization of the phosphorylated form of microsomal glucose-6-phosphatase. Biochem Biophys Res Commun 1969 ; 36 : 119-25.

11. Countaway JL, Waddell ID, Burchell A, Arion WJ. The phosphohydrolase component of the hepatic microsomal glucose-6-phosphatase system is a 36.5-kilodalton polypeptide. I Biol Chem $1988 ; 263$ : 2673-8. les dérivées du réticulum endoplasmique. Elle offre également la possibilité d'étudier cette enzyme sans que les autres phosphatases de la cellule n'interfèrent. Enfin, elle pose le problème du transport du G6P dans les microsomes et représente donc un défi intéressant pour comprendre le fonctionnement et la régulation de la G6Pase.

\section{Modèles de la glucose 6-phosphatase}

La figure 1 montre deux des modèles les plus courants proposés comme l'organisation moléculaire du système G6Pase dans les microsomes. Les deux modèles prévoient que l'hydrolyse du glucose 6-phosphate (G6P) mène à la production de glucose (G1c) et de phosphate (Pi) dans l'espace intramicrosomal (correspondant à la lumière du réticulum endoplasmique) et que leur transport vers le cytosol (milieu extramicrosomal) soit réalisé par des transporteurs distincts pour le phosphate (T2) et pour le glucose (T3). Le modele de transport du substrat est basé sur les postulats suivants [2]. (i) La sous-unité catalytique (E) de la G6Pase est une phosphohydrolase non spécifique, localisée sur la face interne de la membrane microsomale. Par conséquent, la membrane empêche l'accès du G6P au site actif de l'enzyme. (ii) Pour expliquer l'hydrolyse du G6P dans des microsomes intacts, les chercheurs ont pris pour acquis que le transporteur $\mathrm{T} 1$ reconnaît spécifiquement le G6P et lui fait traverser la membrane pour lui permettre l'accès au site actif et que, (iii) le transport de G6P par T1 est l'étape limitante de l'hydrolyse du G6P par la G6Pase.

Quelques propriétés cinétiques du système G6Pase donnent apparemment crédit au modele de transport $d u$ substrat. Le traitement des microsomes avec des détergents augmente, en éliminant la barrière membranaire, l'activité de la G6Pase (concept de la latence) en accord avec les postulats (i) et (iii). On observe d'ailleurs une perte de spécificité de l'enzyme pour le G6P dans des vésicules perméabilisées, conformément aux postulats (i) et (ii). La présence de G6P libre à l'intérieur des microsomes n'a cependant jamais été démontrée bien que la perméabilité des microsomes intacts au G6P ait été indirectement suggérée au moyen de la technique de diffraction de la lumière [3]. Finalement, comme le faisait remarquer Nilsson et coll. [4] : "... la preuve définitive viendra soit de l'isolement et de la reconstitution du transporteur du G6P, soit de la mesure directe de G6P à l'intérieur des vésicules microsomales". Aucune de ces

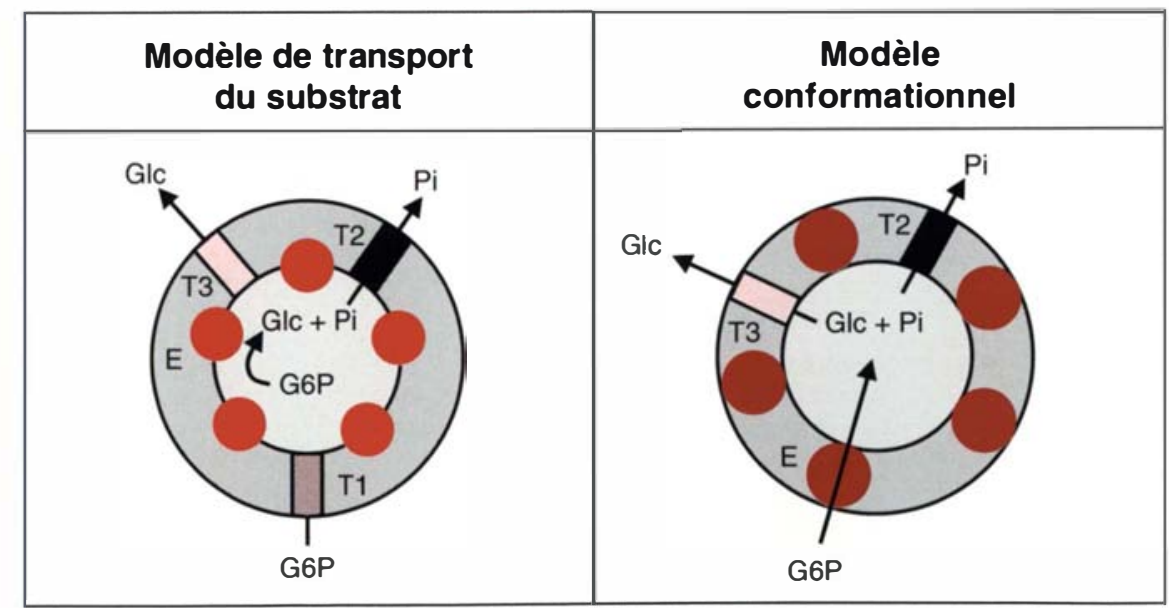

Figure 1. Représentation schématique des deux modèles proposés pour la topologie du système glucose 6-phosphatase dans les microsomes de foie. Le modèle du transport de substrat propose que l'entrée du G6P (T1) dans les microsomes et son hydrolyse (E) sont réalisées par deux protéines différentes tandis qu'une seule protéine (E) est responsable de ces deux fonctions dans le modèle conformationnel. Les deux modèles proposent deux protéines distinctes pour l'exportation du phosphate (T2) et du glucose (T3) hors des microsomes. 


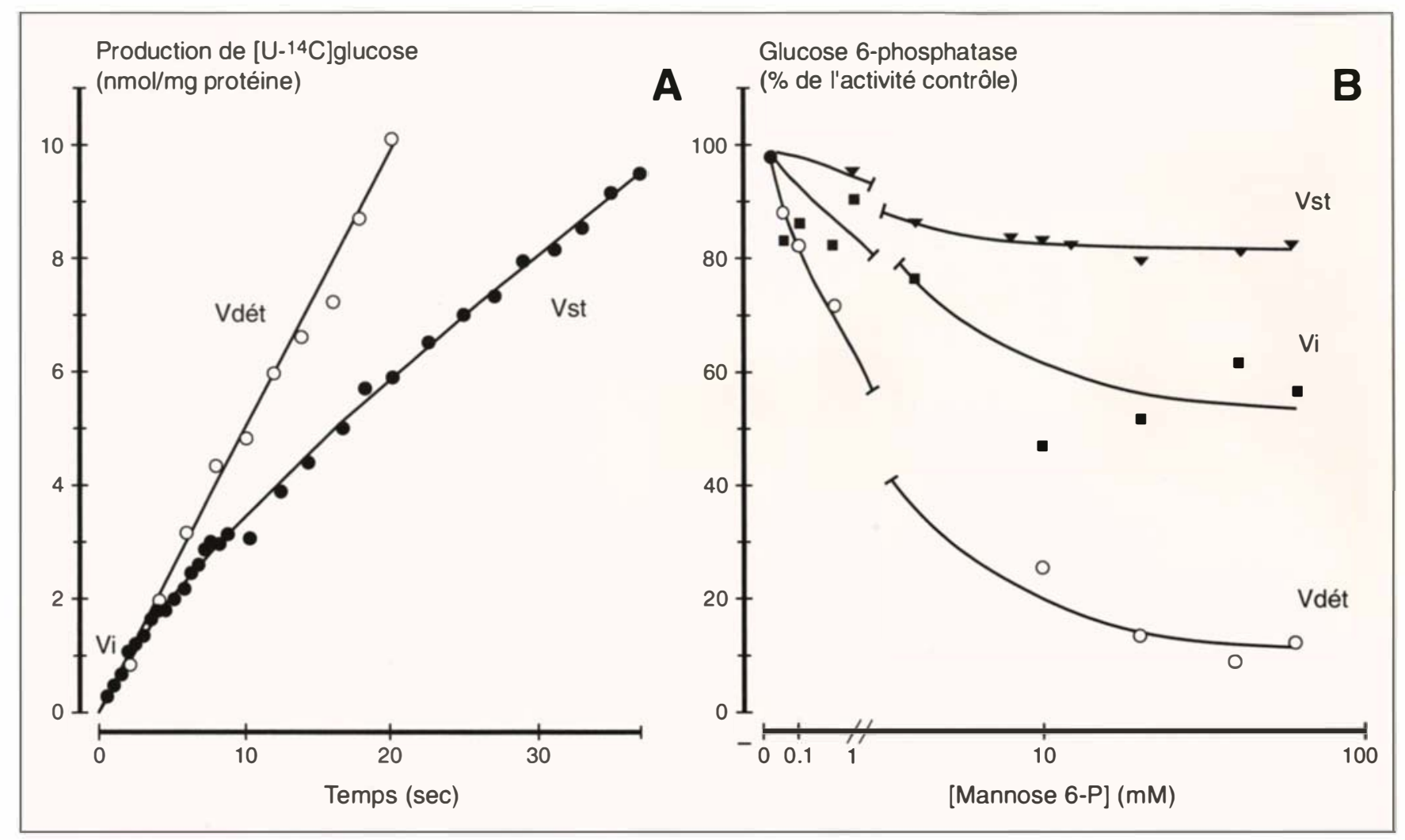

Figure 2A. Formation du [U-14C]glucose à partir de [U-14C]G6P dans les microsomes en fonction du temps. (Figure adaptée de [12]). L'activité de la G6Pase a été mesurée dans des microsomes intacts (symboles fermés) ou dans des microsomes traités au désoxycholate (symboles ouverts) incubés à $30{ }^{\circ} \mathrm{C}$ en présence de $0,1 \mathrm{mM}$ $\left[U-{ }^{14} \mathrm{C}\right]$ G6P au moyen de l'appareil à filtration rapide [12]. Vi et Vst représentent respectivement la vitesse initiale et stationnaire de production de glucose dans des microsomes intacts. Vdét indique la vitesse de production de glucose dans des vésicules traitées avec du désoxycholate $0,4 \%$.

Figure 2B. Inhibition par le M6P de la production de [U-14C]glucose à partir de [U-14C]G6P dans les microsomes. (Figure adaptée de [15]). Les paramètres Vi (carrés fermés), Vst (triangles fermés) et Vdét (ronds ouverts) ont été déterminés au cours d'une incubation en présence de $\left[U-{ }^{14} \mathrm{C}\right] \mathrm{G} 6 \mathrm{P}$, comme décrit dans la légende de la figure $2 A$, en absence ou en présence des concentrations indiquées de M6P non radioactif.

expériences n'a été réalisée jusqu'à présent.

Le modele conformationnel propose que la G6Pase puisse exister sous différents états conformationnels avec des propriétés cinétiques distinctes. Par modification de sa conformation dans la membrane pendant la catalyse, la G6Pase serait capable de fixer son substrat du côté cytosolique des microsomes et de libérer les produits de la réaction dans la lumière. D'après ce modèle, le traitement par un détergent entraînerait une augmentation de l'activité de la G6Pase en modifiant les interactions entre l'enzyme et certains constituants lipidiques (phospholipides) de la membrane indispensables au fonctionnement normal de la G6Pase [1, 5-9]. La réaction catalysée par la G6Pase se caractérise par la formation tran$\mathrm{m} / \mathrm{s} n^{\circ} 5$ ool. 9, mai 93 sitoire d'un complexe enzymephosphate (E-P) avec formation d'une liaison covalente entre le groupe imidazole d'un résidu histidine (N-3) de l'enzyme et le phosphate du G6P [10]. En utilisant cette propriété, il a été possible de marquer la G6Pase avec du $\left[{ }^{32} \mathrm{P}\right] \mathrm{G} 6 \mathrm{P}$ et de montrer que la masse moléculaire devait être environ 36500 [11]. D'autres auteurs [8] ont trouvé, par la technique d'irradiation-inactivation sur des microsomes intacts, que la G6Pase in situ dans la membrane pourrait correspondre à une protéine de $M_{r}$, environ 75000 , qui pourrait être un dimère formé de deux sous-unités de 36000 , liées par des ponts disulfures. La formation d'un dimère de G6Pase ouvre la possibilité d'un contrôle allostérique qui pourrait jouer un rôle dans la latence.

\section{Réévaluation des modèles de la glucose 6-phosphatase}

Un nouveau concept du phénomène de latence

En utilisant un appareil d'échantillonnage et de filtration rapide, nous avons montré que les cinétiques rapides de la G6Pase sont caractérisées par une surproduction temporaire ("burst ") de glucose - et de phosphate - par des microsomes intacts [12] (figure $2 A$, symboles fermés). La solubilisation de la membrane microsomale à l'aide d'un détergent (désoxycholate) entraîne la perte de cette propriété (symboles ouverts). De plus, les vitesses initiales d'hydrolyse du G6P sont les mêmes dans des microsomes intacts 


\section{RÉFÉRENCES}

12. Berteloot $A$, Vidal $H$, van de Werve $G$ Rapid kinetics of liver microsomal glucose-6-phosphatase. Evidence for tightcoupling between glucose-6-phosphate transport and phosphohydrolase activity. $J$ Biol Chem 1991; 266 : 5497-507

13. Arion $\mathrm{WJ}, \mathrm{Wallin} \mathrm{BK}$, Carlson $\mathrm{PW}$, Lange AJ. The specificity of glucose-6-phosphatase of intact liver microsomes. J Biol Chem 1972 ; 247 : 2558-65.

14. Arion WJ, Ballas LM, Lange AJ, Wallim BK. Microsomal membrane permeability and the hepatic glucose-6-phosphatase system. J Biol Chem 1976 ; 251 : 4901-7.

15. Vidal $\mathrm{H}$, Berteloot $\mathrm{A}$, Larue $\mathrm{MJ}, \mathrm{St}$ Denis JF, van de Werve G. Interaction of mannose-6-phosphate with the hysteretic transition in glucose-6-phosphate hydrolysis in intact liver microsomes. FEBS Lett 1992 ; 302 : 197-200.

16. Thorens B, Flier JS, Lodish HF, Kahn BB. Differential regulation of 2 glucose transporters in rat liver by fasting and refeeding and by diabetes and insulin treatment. Diabetes 1990 ; 39 : 712-9.

17. Thorens B, Cheng ZQ, Brown D, Lodish HF. Liver glucose transporter. A basolateral protein in hepatocytes and intestine and kidney cells. Am J Physiol 1990 ; 259 : C279-C85.

18. Craik JD, Elliott KRF. Kinetics of 3-O methylglucose transport in isolated rat hepatocytes. Biochem J 1979 ; 182 : 503-8.

19. Wheeler TJ, Hinkle PC. The glucose transporter of mammalian cells. Annu Rev Physiol 1985 ; 47 : 503-17.

20. Hah J, Jo I, Chakrabarti R, Jung CY Demonstration of a $n$ insulin-insensitive storage pool of glucose transporters in rat hepatocytes and HepG2 cells. J Cell Physiol $1992: 152$ : 56-63.

21. Ciaraldi TP, Horuk R, Matthaei S. Biochemical characterization of the rat liver glucose-transport system. Comparisons with the adipocyte glucose-transport system. Biochem J $1986 ; 240: 115-23$.

22. Waddell ID, Scott H, Grant A, Burchell A. Identification and characterization of a hepatic microsomal glucose transport protein. T3 of the glucose-6-phosphatase system ? Biochem J 1991 ; 275 : 363-7.

23. Waddell ID, Zomerschoe AG, Voice MW, Burchell A. Cloning and expression of a hepatic microsomal glucose transport protein. Comparison with liver plasmamembrane glucose-transport protien GLUT2. Biochem J $1992 ; 286$ : 173-7.

24. Newgard CB, Foster W, McGarry JD. Evidence for suppression of hepatic glucose-6-phosphatase with carbohydrate fee-
(Vi : vitesse initiale de la surproduction) et dans des vésicules traitées au détergent (Vdét). Cette égalité indique que le G6P a un accès similaire au site actif de la G6Pase dans des microsomes intacts et perméabilisés pendant les premières secondes d'incubation. Toutefois, pendant cette période, l'activité de la G6Pase décroît dans les microsomes intacts pour atteindre une vitesse stationnaire plus faible (Vst). A partir de ces résultats, nous avons conclu i) que la latence de la G6Pase dans les microsomes intacts est compatible avec le concept d'une lente transition hystérétique, ii) que la solubilisation de la membrane microsomale à l'aide de détergent empêche cette transition et iii) que le transport de G6P n'est pas restrictif dans les microsomes intacts.

\section{Spécificité de la glucose 6-phospha- tase}

Les résultats rapportés ci-dessus suggèrent l'existence d'une conformation particulière de la G6Pase au début de la réaction catalytique qui aurait des caractéristiques cinétiques proches de la forme obtenue après traitement par un détergent. Pour vérifier cette hypothèse, nous avons étudié la spécificité pour le G6P des différentes formes cinétiques de la G6Pase par la mesure de la vitesse de production de $\left[\mathrm{U}-{ }^{14} \mathrm{C}\right] \mathrm{glucose}$ à partir de $\left[\mathrm{U}-{ }^{14} \mathrm{C}\right] \mathrm{G} 6 \mathrm{P}$ dans des microsomes intacts ( $\mathrm{Vi}$ et $\mathrm{Vst}$ ) et dans des vésicules perméabilisées au détergent (Vdét), en absence ou en présence de mannose 6-phosphate non radioactif (M6P). La figure $2 B$ montre que le M6P n'a que peu d'effet sur Vst mais inhibe presque complètement Vdét, en accord avec des travaux antérieurs $[13,14]$. Le résultat important de cette expérience est que la vitesse initiale de la surproduction de glucose (Vi) dans les microsomes intacts est aussi inhibée par le M6P [15]. Le M6P a donc accès au site actif de la G6Pase dans des vésicules intacts. Ce résultat appuie notre conclusion précédente qui énonce qu'une forme cinétique de l'enzyme, similaire à celle des vésicules traitées au désoxycholate, est présente dans les microsomes intacts au début de l'hydrolyse du G6P et que le transport spécifique du substrat ne peut être l'étape limitante de l'hydrolyse du G6P [12].

Ces résultats démontrent : i) que la latence apparente de la G6Pase dans des microsomes intacts ne peut s'expliquer par un transport limitant dans le compartiment vésiculaire [15]; ii) que cette latence dépend d'une phase transitoire du mécanisme moléculaire d'hydrolyse et que cette transition est réversible, iii) que la liaison du substrat aux microsomes intacts implique une forme libre de l'enzyme avec des propriétés cinétiques similaires à celles de l'enzyme de microsomes traités au détergent, incluant une absence de spécificité pour le G6P ; iv) qu'une liaison ultérieure du ligand doit s'effectuer sur d'autres états conformationnels de la protéine qui seraient induits par suite de la liaison du ligand et/ou de la formation du complexe protéine-phosphate pendant le premier renouvellement de l'enzyme ; et v) que ce second état conformationnel garde en mémoire le premier substrat lié puisque le changement de conformation peut être induit par le M6P sans que celui-ci ne soit hydrolysé par la suite.

\section{Transport de glucose dans le RE}

Deux types principaux de transporteurs glucose sont représentés dans la membrane plasmique des hépatocytes : les formes " érythroïde/cerveau " (GLUT1) et " foie " (GLUT2). La seconde forme est la plus abondante (90-99 \%) et est localisée dans la membrane plasmique sinusoïdale de l'hépatocyte [16, 17]. Elle a un $\mathrm{Km}$ plus élevé pour le glucose (15-20 $\mathrm{mM})$ [18] que la forme GLUT1 (Km 1-2 mM) [19]. Une faible fraction de la réserve de GLUT2 (15\% des transporteurs glucose de l'hépatocyte) pourrait être intracellulaire [20] mais, contrairement au muscle et au tissu adipeux où l'insuline peut mobiliser une réserve intracellulaire de transporteurs (GLUT4), le transport de glucose n'est pas sensible à l'insuline dans le foie [21]. Récemment, Waddell et al. [22] ont identifié un nouveau transporteur de glucose de $52 \mathrm{KDa}$ dans les microsomes de foie de rat. Ce transporteur, dénommé GLUT7, a été purifié puis 


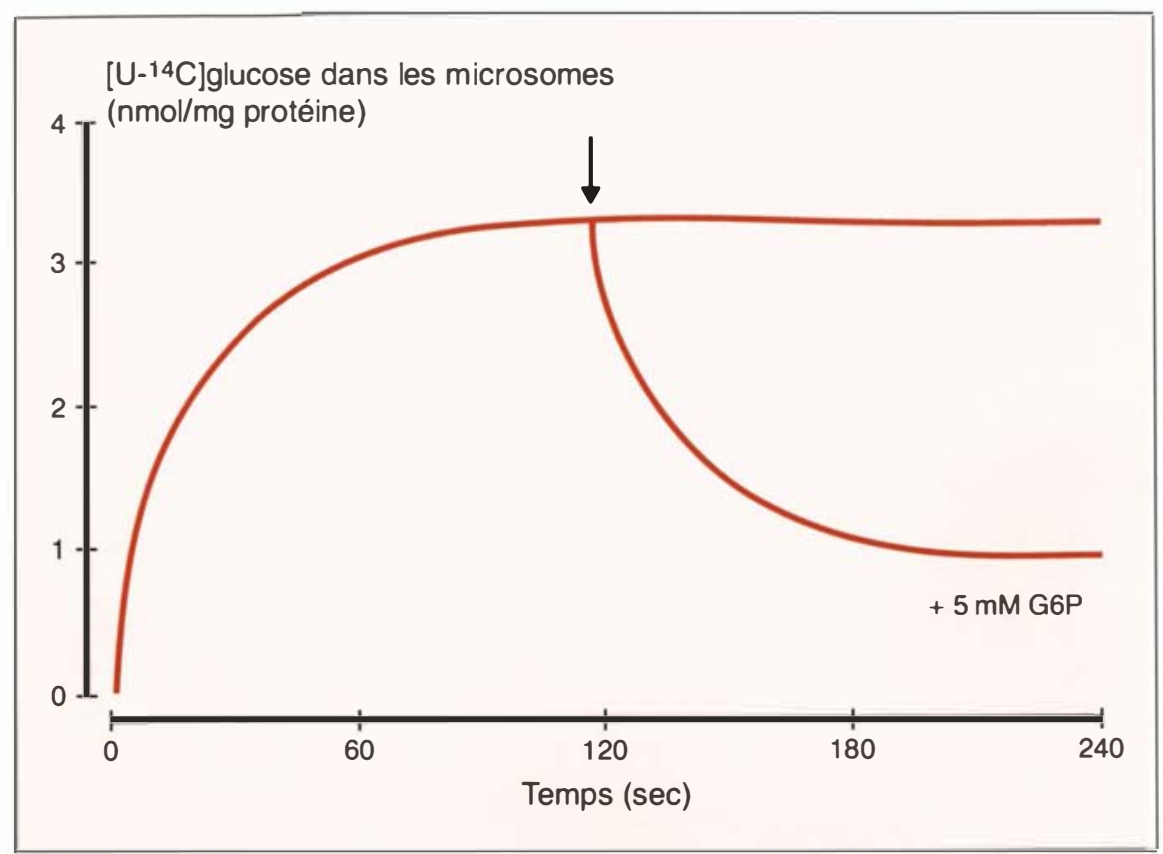

Figure 3. Accumulation de [U-14C]glucose dans les microsomes en fonction du temps. Les microsomes ont été incubés à $30^{\circ} \mathrm{C}$ en présence de $0,2 \mathrm{mM}\left[U-{ }^{14} \mathrm{C}\right] \mathrm{G} 6 \mathrm{P}$ au moyen de l'appareil à filtration rapide [12]. L'addition d'un excès de G6P non marqué (5 $\mathrm{mM}$ ) après deux minutes d'incubation est indiquée par la flèche.

cloné et sa séquence déduite de l'ADN complémentaire correspondant [23]. Il présente $68 \%$ d'identité avec GLUT2. L'expression de GLUT7 dans des cellules COS 7 a permis de montrer une localisation subcellulaire de la protéine au sein du réticulum endoplasmique [23]. La question qui se pose alors consiste à savoir si GLUT7 correspond au transporteur T3 des modèles proposés à la figure 1. Nous avons montré que le glucose radioactif provenant de l'hydrolyse de $\left[\mathrm{U}-{ }^{14} \mathrm{C}\right] \mathrm{G} 6 \mathrm{P}$ s'accumule dans les microsomes de foie de rat, pour atteindre un plateau (figure 3) alors que la production totale de glucose se poursuit à vitesse constante (Vst) (voir figure 2A). Dans ces conditions, la vitesse d'hydrolyse du G6P doit donc être égale à la vitesse de sortie du glucose des vésicules [12]. Comme le montrent la figure 3 et un travail précédent [22], la valeur de ce plateau diminue lorsqu'un excès de G6P non radioactif est ajouté, indiquant ainsi qu'à l'équilibre d'accumulation, l'hydrolyse du G6P et la production de glucose dans la lumière des microsomes est suivi d'un efflux de glucose des vésicules. Aucune capture de radioactivité $\mathrm{m} / \mathrm{s} n^{\circ} 5$ vol. 9, mai 93
(2-5 mM) [14] est plus élevé que le contenu intracellulaire en G6P (70-500 mmol/g foie). Le flux de substrat qu'emprunte la voie de la G6Pase n'est cependant pas toujours compatible avec les concentrations de $\mathrm{G} 6 \mathrm{P}$, en particulier lorsque le rapport insuline/glucagon est modifié [24, 25]. Les hormones pourraient donc moduler la G6Pase par un mécanisme " on/off " qui s'ajouterait à leurs effets sur la disponibilité en substrat. L'inhibition de la G6Pase microsomale par un phosphoinositol glycan insulino-mimétique a récemment été rapportée [26]. La possibilité d'une régulation hormonale de la G6Pase par phosphorylation/déphosphorylation reste incertaine [27-30]. Une inhibition par le glycogène de la G6Pase a été décrite brièvement récemment [31]. La déficience en insuline obtenue à la suite d'un jeûne prolongé ou par un diabète expérimental augmente l'activité de la G6Pase et induit la synthèse de novo de l'enzyme, et probablement la synthèse de la sous-unité catalytique (E dans la figure 1), parce que cette augmentation est plus évidente dans des microsomes perméabilisés à l'aide d'un détergent que dans des vésicules intactes (la latence apparente est augmentée). L'administration de glucocorticoïdes à des rats augmente la G6Pase de foie dans les microsomes intacts plutôt que perméabilisés (la latence apparente est diminuée), ce qui mène à l'hypothèse que la putative translocase $\mathrm{T} 1$ est augmentée plutôt que E (revu dans [32]), mais la formation de sous-unités de l'unité catalytique altérées par les glucocorticoïdes a également été proposée [33]. A la lumière de la réévaluation du concept de la latence que nous avons discuté précédemment, ces altérations hormonales devraient être étudiées par la mesure des cinétiques rapides de la G6Pase et par le clonage de cette enzyme qui devrait permettre d'identifier les éléments de contrôle du promoteur et d'obtenir une sonde spécifique pour mesurèr les niveaux d'ARN messager dans ces différentes conditions.

\section{Déficits du système glucose 6-phosphatase}

Les glycogénoses de type 1 sont cau- 


\section{RÉFÉRENCES}

25. Christ B, Probst I, Jungermann K. Antagonistic regulation of the glucose/glucose 6-phosphate cycle by insulin and glucagon in culturel hepatocytes. Biochem $J$ $1986 ; 238: 185-91$.

26. Misek DE, Saltiel AR. An inositol phosphate glycan derived from a Trypanosoma brucei glycosyl-phosphatidylinositol mimics some of the metabolic actions of insulin. J Biol Chem 1992 ; 267 : 16266-73.

27. Burchell A, Burchell B. Stabilization of partially-purified glucose-6-phosphatase by fluoride. Is enzyme inactivation caused by dephosphorylation? FEBS Lett $1980 ; 118$ : 180-4.

28. Begley PJ, Craft JA. Evidence for protein phosophyration as a regulatory mechanism for hepatic microsomal glucose-6-phosphatase. Biochem Biophys Res Commun 1981; 103 : 1029-34.

29. Burchell A, Burchell B, Arion WJ, Walls HE. A critical evaluation of the possible modulation of hepatic microsomal glucose-6-phosphatase activity by protein phosphorylation. Biochem Biophys Res Commun $1982 ; 107$ : 1046-52.

30. Singh J, Martin RE, Nordlie RC. Glucose-6-phosphatase : is activity regulated by phosphorylation-dephosphorylation ? Can $J$ Biochem Cell Biol 1983 ; 61 : 1085-9.

31. Liu Z, Gardner L, Barrett E. Regulation of glucose-6-phosphatase by glycogen. Diabetes 1992 ; 41 : 163A.

32. Sukalski KA, Nordlie RC. Glucose-6phosphatase : two concepts of membranefunction relationship. Adv Enzymol Relat Areas Mol Biol 1989 ; 62 : 93-117.

33. Traxinger R R, Nordlie RC. Hormonal responses of glucose-6-phosphatase catalytic unit studied by stopped-flow analysis. Biochem Cell Biol 1990; 68 : 454-8.

34. Dreyfus JC. Une glycogénose complexe: le déficit du système glucose6-phosphatase. médecine-sciences $1989 ; 9$ : 692-3.

35. Schaub J, Heyne K. Glycogen storage disease type 1b. Eur J Pediatr $1983 ; 140$ : 283-88.

36. Burchell A. Molecular pathology of glucose-6-phosphatase. FASEB J 1990 ; 4 : 2978-88.

37. de Duve C, Berthet J, Hers HG, Dupret L. Le système hexose-phosphatasique. 1. Existence d'une glucose-6-phosphatase spécifique dans le foie. Bull Sté Chim Biol 1949 ; 31 : 1242-53. sées par des déficiences génétiques transmises comme des caractères autosomiques récessifs $\left(\mathrm{m} / \mathrm{s} \quad n^{\circ} 9\right.$, p. 692, 1989 [34]). La forme 1a (absence de l'activité G6Pase dans des microsomes intacts et perméabilisés) devrait permettre de mettre en évidence un transporteur $\mathrm{T} 1$ pour le G6P, qui serait distinct de la composante hydrolase du système glucose 6-phosphatase selon le modèle de transport du substrat (figure 1). Nous avons récemment étudié le fonctionnement de la G6Pase dans des microsomes de foie d'une patiente atteinte de glycogénose de type 1a. Nous n'avons pas pu mettre en évidence une accumulation de radioactivité dans les vésicules en présence de $\left[\mathrm{U}-{ }^{14} \mathrm{C}\right] \mathrm{G} 6 \mathrm{P}$ (résultats non publiés), suggérant l'absence de transport de G6P dans ces microsomes. Ce résultat est en accord avec notre hypothèse soit que la G6Pase peut reconnaître et lier son substrat sur la face externe des microsomes [12]. La forme $1 b$ est caractérisée par une G6Pase hépatique non fonctionnelle in vivo mais active dans des microsomes perméabilisés. Elle a été expliquée par l'absence de T1 parce que la G6Pase est inactive dans des microsomes intacts mais manifeste une activité normale dans des vésicules traitées au détergent $[1,35,36]$. Alternativement, la glycogénose de type $1 \mathrm{~b}$ pourrait être due à une mutation de la protéine G6Pase qui affecterait ses propriétés, particulièrement son interaction avec les lipides de la membrane du réticulum endoplasmique.

\section{Conclusions}

Plus de 40 ans après la découverte de la G6Pase [37], le mécanisme d'action de cette enzyme qui permet la production de glucose par le foie n'est pas encore bien compris. Un débat reste ouvert quant à l'existence d'une protéine spécifique transportant le substrat G6P (T1). Ce transporteur ou alors un changement de conformation de la G6Pase, que nos résultats favorisent, pourrait exercer son rôle physiologique lors des modifications de l'état hormonal ainsi que dans des pathologies liées à une déficience du système G6Pase. Les autres transporteurs putatifs du complexe enzymatique (T2 ou T3) doivent encore être caractérisés. Le contrôle de l'expression de la G6Pase, que l'on trouve uniquement dans les tissus hépatique et rénal, reste encore à découvrir. La recherche sur la G6Pase doit donc faire appel maintenant aux techniques de la biologie moléculaire. L'obtention d'une sonde spécifique et le clonage d'un ADN complémentaire codant pour la G6Pase marqueront probablement les prochaines étapes significatives à franchir. A partir de là, il sera possible d'établir les preuves définitives concernant l'organisation de la G6Pase dans la membrane, de mener à terme l'étude de la régulation de son expression dans les cellules, ainsi que l'analyse des glycogénose de type 1 à l'échelle moléculaire

\section{Summary}

The hepatic glucose 6-phosphatase system : components, kinetic properties, regulation and defects

Glucose 6-phosphatase (G6Pase) catalyzes the last step leading to liver glucose production and is therefore essential to maintain normoglycemia. Given the strategic location of this enzyme at the cross-roads of gluconeogenesis, glycogenolysis and glycolysis, as well as the severe metabolic disorders resulting from different forms of inherited G6Pase deficiency (glycogenosis type 1), studies on the structure-function relationship and regulation of the enzyme are essential to understand glucose metabolism in vivo. In this review, two models explaining the molecular organization of the G6Pase system are discussed, the conformational model and the substrate transport model, as well as the hormonal regulation of G6Pase and the molecular basis of its pathological deficiency. Our results based on rapid kinetics and specificity of the enzyme for its substrate are in favor of the conformational model. An unidirectional transport of glucose produced by the hydrolysis of $\mathrm{G} 6 \mathrm{P}$ has also been revealed in microsomes.

\section{TIRÉS A PART}

G. van de Werve. 